\title{
Structural Dependence of Reductive Defluorination of Linear PFAS Compounds in a
}

\section{UV/Electrochemical System}

\author{
Unnati Rao a,\#, Yiming Sü ${ }^{a, \#, ~ C h i a ~ M i a n g ~ K h o r a, ~ B o n g y e o n ~ J u n g a, ~ S h e n g c u n ~ M a a, ~ D a v i d ~ M . ~ C w i e r t n y ~}{ }^{b}$,
} Bryan M. Wong', David Jassbya ${ }^{*}$

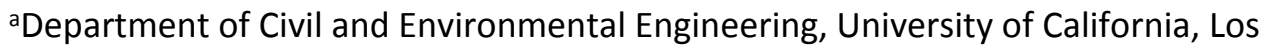
Angeles, California 90095-153, United States

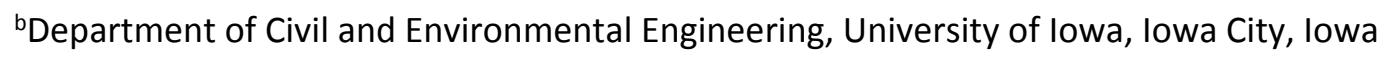
52242, United States

'Department of Chemical \& Environmental Engineering, Materials Science \& Engineering Program, University of California, Riverside, California, 92521, United States

* Corresponding Author, jassby@ucla.edu

0

1

2

3

24




\section{Supporting information}

Contents:

27 Table S1: Surface coverage on the electrode (measured by XPS).

28 Equations S1-S5: Theoretical calculations comparing electron transfer rates from the .53

29 electrode to a sorbed PFOS molecule with and without applied potential

Table S2: Marcus theory parameters for the various reactions when the

31 (CNT + ammonium) complex has a different charged state.

32 Figure S1: Schematic depicting the relationships between $\Delta \mathrm{G}^{\wedge}, \llbracket \Delta \mathrm{G} \rrbracket^{\wedge} \ddagger$, and. $\lambda$ for an electron transfer between two free energy curves of a reactant and product.

34 The dotted diabatic curves are allowed to intersect whereas the solid-colored adiabatic

35 curves avoid crossing

37 degradation of (c) TCE+TB and (d) CDCE+TB under UV/2V, UV/OV and UV only. 
50 Table S1: Surface coverage on the electrode (measured by XPS)

\begin{tabular}{|cc|}
\hline Species & Fluoride surface coverage \\
\hline PFNA & $0.19 \pm 0.06 \%$ \\
\hline PFOA & $0.13 \pm 0.01 \%$ \\
\hline PFHpA & $0.08 \pm 0.04 \%$ \\
\hline PFOS & $4.45 \pm 0.49 \%$ \\
\hline PFHxS & $0.65 \pm 0.23 \%$ \\
\hline PFBS & below detection limit \\
\hline
\end{tabular}

51

52 Theoretical calculations comparing electron transfer rates from the electrode to a sorbed PFOS molecule with and without applied potential

54 To calculate and predict the electron-transfer rates for all the chemical species examined in this work, we utilized Marcus theory, ${ }^{1,2}$ which describes the electron-transfer process between a donor and acceptor

56 molecule according to the following general equation:

$$
D^{m}+A^{n_{k_{E T}}} D^{m+1}+A^{n-1}
$$

57 On the left-hand side of Eq. (1), $D^{m}$ denotes a donor molecule with an electronic charge state of $m$, and $A^{n}$

58 represents an acceptor molecule having a charge state of $n$ (we have chosen to use this notation since 59 many of the chemical species studied in this work have various electronic charged states). After the 60 electron-transfer process has occurred, the donor $\left(D^{m+1}\right)$ on the right-hand side of Eq. (1) now has a charge 61 state of $m+1$, and the acceptor $\left(A^{n-1}\right)$ gains an electron and attains a charge state of $n-1$. The non62 adiabatic electron-transfer rate, $k_{\mathrm{ET}}$, in Marcus theory is given by 


$$
k_{E T}=\frac{4 \pi}{h} V_{r p}^{2}\left(\frac{1}{4 \pi \lambda k_{B} T}\right)^{\frac{1}{2}} \exp \left[-\frac{\Delta G^{\ddagger}}{k_{B} T}\right]
$$

63 where $h$ is Planck's constant, $k_{B}$ is Boltzmann's constant, $T$ is the temperature, $V_{r p}$ is the transfer integral

64 between the reactant and product, $\lambda$ is the reorganization energy, and $\Delta G^{\ddagger}$ is the activation energy $65\left(=\frac{\left(\lambda+\Delta G^{\circ}\right)^{2}}{4 \lambda}\right)$, where $\Delta G^{\circ}$ is the difference in energy between the reactants and products in Eq. (1). Figure 66 S1 depicts the relationships between $\Delta G^{\circ}, \Delta G^{\ddagger}$, and $\lambda$ for two Marcus-theory parabolas that represent 67 the free energy curves of the reactant and product states. In the diabatic (i.e., non-adiabatic) representation, the electronic character of the reactant and product does not change as one moves along

69 the reaction coordinate; hence, the reactant and product curves (colored red and blue) cross with an 70 activation energy of $\Delta G^{\ddagger}$. In contrast, within the adiabatic representation (which are 71 solutions/eigenstates of the Schrödinger equation and can be directly computed with quantum chemistry 72 methods), the two curves avoid crossing and form an upper and lower curve (colored in solid black).

73 As mentioned previously, quantum chemistry methods can be used to directly calculate $\Delta G^{\circ}, \lambda$, and $V_{r p}$ to 74 yield the electron-transfer rate, $k_{\mathrm{ET}}$. For generality, we use the standard notation $E(a \mid b)$ to represent the 75 energy of state " $a$ " calculated at the optimized equilibrium structure of state " $b$ " (i.e., $a$ and $b$ may or may 76 not be the same). To this end, the difference in energy between the reactants and products, $\Delta G^{\circ}$, is given 77 by

$$
\Delta G^{\circ}=E\left(D^{m} \mid D^{m}\right)+E\left(A^{n} \mid A^{n}\right)-E\left(D^{m+1} \mid D^{m+1}\right)-E\left(A^{n-1} \mid A^{n-1}\right) .
$$

78 The reorganization energy can be computed with the conventional "four-point" method":

$$
\lambda=E\left(D^{m+1} \mid D^{m}\right)-E\left(D^{m+1} \mid D^{m+1}\right)+E\left(A^{n-1} \mid A^{n}\right)-E\left(A^{n-1} \mid A^{n-1}\right) .
$$

79 Finally, the transfer integral for electrons, $V_{r p}$, can be approximated by an energy-splitting expression ${ }^{4}$ 80 given by 


$$
V_{r p}=\frac{\varepsilon_{\mathrm{LUMO}+1}-\varepsilon_{\mathrm{LUMO}}}{2}
$$

81 where $\varepsilon_{\mathrm{LUMO}}$ and $\varepsilon_{\mathrm{LUMO}}+1$ are the lowest unoccupied molecular orbital (LUMO) and the (L+1)UMO orbital

82 energies of the dimer complex. With the adiabatic quantities in Eqs. (3) - (5) properly defined (and

83 computable with quantum chemistry methods), this allow us to calculate the non-adiabatic electron-

84 transfer rate, $k_{\mathrm{ET}}$, in Eq. (2). All of the parameters in Eqs. (3) - (5) were obtained at the B97D/6-31G(d,p)

85 level of theory in conjunction with a conductor-like polarizable continuum model (PCM) devised by Tomasi

86 and co-workers ${ }^{5-9}$, which we have previously used to calculate radical-based oxidation reactions in other

87 aqueous systems. ${ }^{10,11}$

88 We used the ET process between a CTAB-functionalized CNT electrode and PFOS as a model calculation.

89 To rationalize the electron-transfer rates between the various charged states of the electrode (CNT +

90 ammonium) and PFOS, we considered two specific reactions:

$$
\begin{aligned}
& \left(\mathrm{CNT}+\text { ammonium }^{+1}+\mathrm{PFOS}^{-1} \stackrel{k_{E T}}{\rightarrow}\left(\mathrm{CNT}+\text { ammonium }^{+2}+\mathrm{PFOS}^{-2},\right.\right. \\
& \left(\mathrm{CNT}+\text { ammonium }^{0}+\mathrm{PFOS}^{-1} \stackrel{k_{E T}}{\rightarrow}\left(\mathrm{CNT}+\text { ammonium }^{+1}+\mathrm{PFOS}^{-2},\right.\right.
\end{aligned}
$$

91 where the subscripts follow the same convention described previously in Eq. (1) and denote the charge

92 state of the specific chemical species/complex. Reactions R1 and R2 were chosen as representative 93 models to contrast electron-transfer rates when the (CNT + ammonium) complex has a different charge

94 state (i.e. the (CNT + ammonium $)^{0}$ complex has an extra negative charge compared to the positively95 charged (CNT + ammonium $)^{+1}$ complex). The following Marcus theory parameters for each of the molecular reactions were obtained at the B97D/6-31G(d,p) level of theory with the PCM model (Table 2):

97 
Table S2: Marcus theory parameters for the various reactions when the (CNT + ammonium) complex has a different charged state.

\begin{tabular}{|ccccc|}
\hline Reaction & $\boldsymbol{V}_{r p}(\mathbf{e V})$ & $\lambda(\mathbf{e V})$ & $\Delta \boldsymbol{G}^{\circ}(\mathbf{e V})$ & $\boldsymbol{k}_{\mathrm{ET}}\left(\mathbf{s}^{-1}\right)$ \\
\hline $\mathbf{R} 1$ & 0.135 & 0.648 & -2.91 & $4.84 \times 10^{-20}$ \\
\hline $\mathbf{R 2}$ & 0.138 & 0.650 & -2.54 & $6.48 \times 10^{-10}$ \\
\hline
\end{tabular}

101

As can be seen in Table 2, the electron-transfer rate of $\mathrm{R} 2$ is significantly faster than $\mathrm{R} 1$, primarily due to the $\Delta G^{\circ}$ term. In other words, the electron-transfer rate from the (CNT + ammonium) complex to PFOS is significantly faster when it is more negatively charged, which is reflected in the $\Delta G^{\circ}$ energetic difference.

Since it is well-known that DFT-predicted rate constants are known to exhibit error, the numbers presented in the table are only meant to give overall trends. While the $\mathrm{k}_{\mathrm{ET}}$ rates reported in Table 2 represent electron-transfer reactions under ideal conditions (i.e., they do not take into account other nonadiabatic electronic/nuclear effects), the significantly larger R2 value clearly indicates that a negatively weakening to a larger extent, thus making them more susceptible to breakdown by hydrated electrons. 


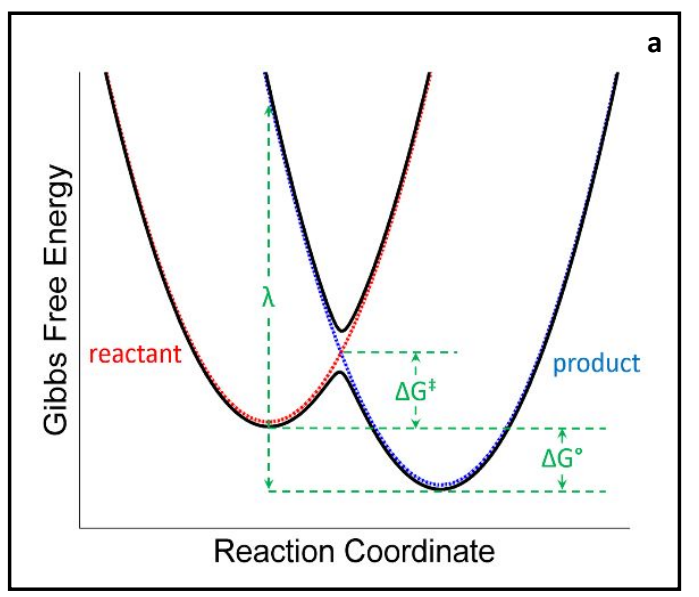

Figure S1: Schematic depicting the relationships between $\Delta \mathrm{G}^{\wedge^{\circ}}, \llbracket \Delta G \rrbracket^{\wedge} \ddagger$, and $\lambda$ for an electron transfer between two free energy curves of a reactant and product. The dotted diabatic curves are allowed to intersect whereas the solid-colored adiabatic curves avoid crossing
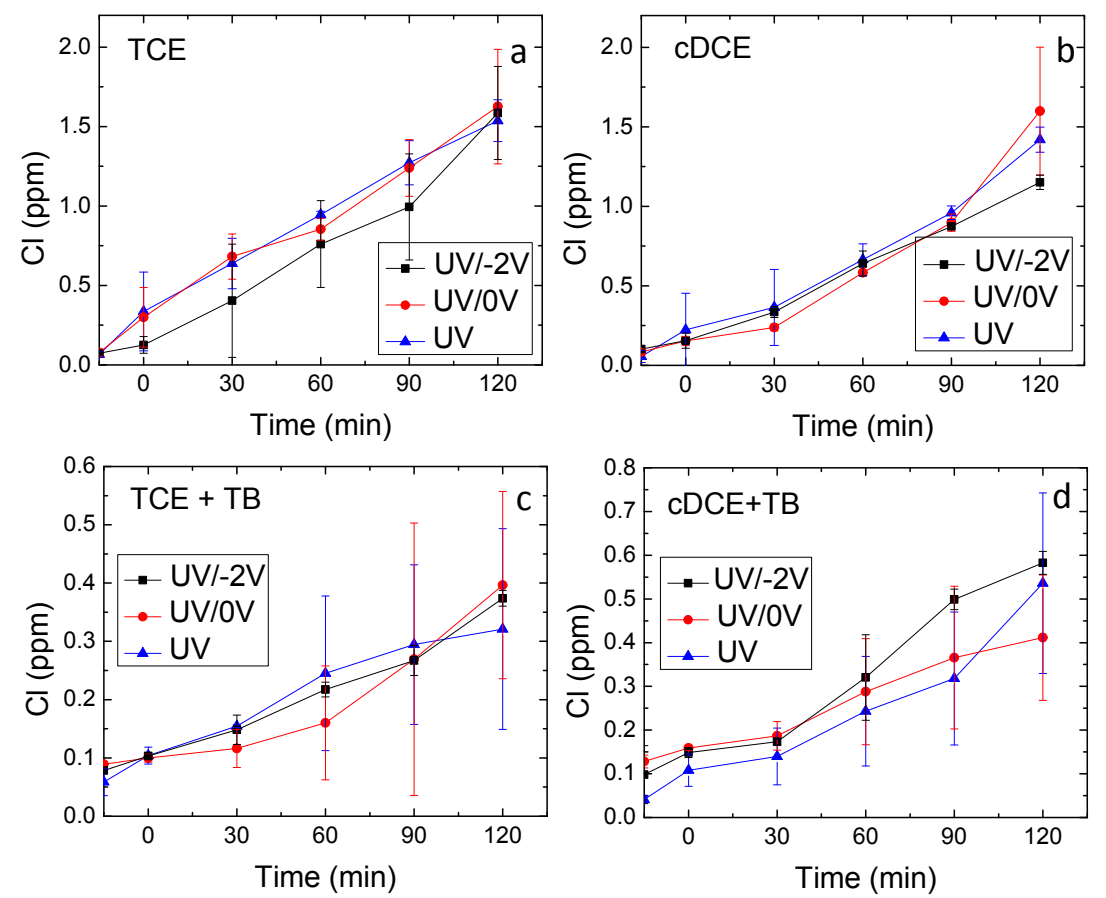

Figure S2: Degradation of (a) TCE and (b) CDCE under UV/-2V, UV/OV and UV only; and degradation of (c) TCE+TB and (d) CDCE+TB under UV/-2V, UV/OV and UV only. 
121 1. Marcus, R. A.; Sutin, N., Electron transfers in chemistry and biology. Biochimica et Biophysica 122 Acta (BBA)-Reviews on Bioenergetics 1985, 811 (3), 265-322.

$1232 . \quad$ Marcus, R. A., Electron transfer reactions in chemistry. Theory and experiment. Rev Mod Phys 124 1993, $65(3), 599-610$.

$1253 . \quad$ Nelsen, S. F.; Blomgren, F., Estimation of Electron Transfer Parameters from AM1 Calculations. 126 The Journal of Organic Chemistry 2001, 66 (20), 6551-6559.

127 4. Newton, M. D., Quantum chemical probes of electron-transfer kinetics: the nature of donor128 acceptor interactions. Chemical Reviews 1991, 91 (5), 767-792.

$129 \quad 5 . \quad$ Fortunelli, A.; Tomasi, J., The Implementation of Density-Functional Theory within the 130 Polarizable Continuum Model for Solvation. Chem Phys Lett 1994, 231 (1), 34-39.

131 6. Tomasi, J.; Bonaccorsi, R.; Cammi, R.; Delvalle, F. J. O., Theoretical Chemistry in Solution - Some 132 Results and Perspectives of the Continuum Methods and in Particular of the Polarizable Continuum 133 Model. Theochem-J Mol Struc 1991, 80, 401-424.

134 7. Delvalle, F. J. O.; Bonaccorsi, R.; Cammi, R.; Tomasi, J., Electron Correlation and Solvation 135 Effects .3. Influence of the Basis Set and the Chemical-Composition on the Solvation Energy Components 136 Evaluated with the Quantum-Mechanical Polarizable Continuum Model. Theochem-J Mol Struc 1991, 76, 137 295-312.

138 8. Delvalle, F. J. O.; Tomasi, J., Electron Correlation and Solvation Effects .1. Basic Formulation and 139 Preliminary Attempt to Include the Electron Correlation in the Quantum-Mechanical Polarizable 140 Continuum Model So as to Study Solvation Phenomena. Chem Phys 1991, 150 (2), 139-150.

141 9. Aguilar, M. A.; Delvalle, F. J. O.; Tomasi, J., Electron Correlation and Solvation Effects .2. The 142 Description of the Vibrational Properties of a Water Molecule in a Dielectric Given by the Application of 143 the Polarizable Continuum Model with Inclusion of Correlation-Effects. Chem Phys 1991, 150 (2), 151144161.

145 10. Pari, S.; Wang, I. A.; Liu, H.; Wong, B. M., Sulfate radical oxidation of aromatic contaminants: a 146 detailed assessment of density functional theory and high-level quantum chemical methods.

147 Environmental Science: Processes \& Impacts 2017, 19 (3), 395-404.

$148 \quad$ 11. Raza, A.; Bardhan, S.; Xu, L.; Yamijala, S. S. R. K. C.; Lian, C.; Kwon, H.; Wong, B. M., A Machine 149 Learning Approach for Predicting Defluorination of Per- and Polyfluoroalkyl Substances (PFAS) for Their 150 Efficient Treatment and Removal. Environmental Science \& Technology Letters 2019, 6 (10), 624-629. 